\title{
Blow-up solutions, global existence, and exponential decay estimates for second order parabolic problems
}

Juntang Ding* and Ming Wang

${ }^{\text {"Correspondence: }}$

djuntang@sxu.edu.cn

School of Mathematical Sciences,

Shanxi University, Taiyuan, 030006,

P.R. China

\section{Springer}

\begin{abstract}
In this paper, we study the blow-up solutions, global existence, and exponential decay estimates for a class of second order parabolic problems with Dirichlet boundary conditions. By constructing auxiliary functions and using maximum principles, the sufficient conditions for the existence of the blow-up solution, the sufficient conditions for the global existence of the solution, an upper bound for the 'blow-up time', and some explicit exponential decay bounds for the solution and its derivatives are specified.
\end{abstract}

MSC: $35 \mathrm{~K} 60 ; 35 \mathrm{~K} 20$

Keywords: blow-up solution; global existence; exponential decay estimate

\section{Introduction}

Many authors have studied the blow-up solutions, global existence, and exponential decay estimates of nonlinear parabolic problems (see, for instance, [1-14]). In this paper, we investigate the following second order parabolic problems with Dirichlet boundary conditions:

$$
\begin{cases}(k(u))_{t}=\nabla \cdot(g(u) \nabla u)+f(u), & (x, t) \in D \times(0, T), \\ u=0, & (x, t) \in \partial D \times(0, T), \\ u(x, 0)=h(x), & x \in \bar{D},\end{cases}
$$

where $D \subset \mathbb{R}^{N}(N \geq 2)$ is a bounded convex domain with smooth boundary $\partial D \in C^{2, \varepsilon}$, $T$ is the maximal existence time of $u$, and $\bar{D}$ is the closure of $D$. Set $\mathbb{R}^{+}:=(0,+\infty)$. We assume, throughout the paper, that $f(s)$ is a nonnegative $C^{1}\left(\mathbb{R}^{+}\right)$function, $f(0)=0, g(s)$ is a positive $C^{2}\left(\mathbb{R}^{+}\right)$function, $g^{\prime}(s) \leq 0$ for any $s \in \mathbb{R}^{+}, k(s)$ is a $C^{2}\left(\overline{\mathbb{R}^{+}}\right)$function, $k^{\prime}(s)>0$ for any $s \in \overline{\mathbb{R}^{+}}$, and $h(x)$ is a nonnegative $C^{2}(\bar{D})$ function, $h(x) \neq \equiv$ for any $x \in \bar{D}$. Under these assumptions, it follows from the maximum principle [15] that $u(x, t)$ is nonnegative.

Some special cases of the problem (1.1) have been discussed already. Payne et al. in [16] dealt with the following problem:

$$
\begin{cases}u_{t}=\Delta u+f(u), & (x, t) \in D \times(0, T), \\ u=0, & (x, t) \in \partial D \times(0, T), \\ u(x, 0)=h(x), & x \in \bar{D} .\end{cases}
$$

(c) 2015 Ding and Wang. This article is distributed under the terms of the Creative Commons Attribution 4.0 International License (http://creativecommons.org/licenses/by/4.0/), which permits unrestricted use, distribution, and reproduction in any medium, provided you give appropriate credit to the original author(s) and the source, provide a link to the Creative Commons license, and indicate if changes were made. 
They established conditions on data sufficient to preclude blow-up and to ensure that the solution and its spatial gradient decay exponentially for all $t>0$. In [17], Enache researched the following problem:

$$
\begin{cases}u_{t}=\nabla \cdot(g(u) \nabla u)+f(u), & (x, t) \in D \times(0, T), \\ u=0, & (x, t) \in \partial D \times(0, T), \\ u(x, 0)=h(x), & x \in \bar{D} .\end{cases}
$$

His purpose was to establish conditions on the data sufficient to guarantee blow-up of solution at some finite time, conditions to ensure that the solution remains bounded as well as conditions to derive some explicit exponential decay bounds for the solution and its derivatives. Some authors also discussed blow-up phenomena for parabolic problems with Dirichlet boundary conditions and obtained a lot of interesting results (see, for instance, [18-24]).

In the process of heat conduction and mass diffusion, many problems can be summarized as the problem (1.1). Therefore, in this paper, we study the problem (1.1). By constructing auxiliary functions and using maximum principles, the sufficient conditions for the existence of the blow-up solution, the sufficient conditions for the global existence of the solution, an upper bound for the 'blow-up time', and some explicit exponential decay bounds for the solution and its derivatives are specified. Our results extend and supplement those obtained in $[16,17]$.

We proceed as follows. In Section 2 we study the blow-up solution of (1.1). Section 3 is devoted to the global solution of (1.1) and the explicit exponential decay bounds for the solution. The explicit exponential decay bounds for the derivatives of the solution are given in Section 4. A few examples are presented in Section 5 to illustrate the applications of the abstract results.

\section{Blow-up solution}

In order to get the sufficient conditions for the existence of the blow-up solution, we define the following functions:

$$
\begin{array}{ll}
F(u):=\int_{0}^{u} f(s) g(s) \mathrm{d} s, & G(u):=2 \int_{0}^{u} s g(s) k^{\prime}(s) \mathrm{d} s, \\
A(t):=\int_{D} G(u(x, t)) \mathrm{d} x, & B(t):=\int_{D}\left(F(u)-\frac{1}{2} g^{2}(u)|\nabla u|^{2}\right) \mathrm{d} x .
\end{array}
$$

The following theorem is the main result for the blow-up solution.

Theorem 2.1 Let u be a classical solution of the problem (1.1). Suppose we have the following.

(i)

$$
\left(g(s) k^{\prime}(s)\right)^{\prime} \leq 0, \quad s f(s) g(s) \geq \frac{1}{2}(4+\alpha) F(s), \quad s \in \mathbb{R}^{+},
$$

where $\alpha$ is a positive constant.

(ii)

$$
\lim _{s \rightarrow 0^{+}} s^{2} g(s)=0
$$


(iii)

$$
B(0)=\int_{D}\left(F(h)-\frac{1}{2} g^{2}(h)|\nabla h|^{2}\right) \mathrm{d} x \geq 0 .
$$

Then $u(x, t)$ must blow up at some finite time $t^{*}<T$ and

$$
T:=\frac{4}{\alpha(\alpha+4)} A(0) B^{-1}(0) \leq+\infty .
$$

Proof Making use of the differential equation (1.1), of the divergence theorem, of the fact that $g^{\prime} \leq 0$ and of the assumption (2.1), we have

$$
\begin{aligned}
A^{\prime}(t) & =2 \int_{D} g k^{\prime} u u_{t} \mathrm{~d} x=2 \int_{D} f g u \mathrm{~d} x+2 \int_{D} g u\left(g \Delta u+g^{\prime}|\nabla u|^{2}\right) \mathrm{d} x \\
& =2 \int_{D} f g u \mathrm{~d} x-2 \int_{D} g g^{\prime} u|\nabla u|^{2} \mathrm{~d} x-2 \int_{D} g^{2}|\nabla u|^{2} \mathrm{~d} x \\
& \geq 2 \int_{D} f g u \mathrm{~d} x-2 \int_{D} g^{2}|\nabla u|^{2} \mathrm{~d} x \\
& \geq(4+\alpha) \int_{D}\left(F(u)-\frac{1}{2} g^{2}(u)|\nabla u|^{2}\right) \mathrm{d} x+\frac{\alpha}{2} \int_{D} g^{2}(u)|\nabla u|^{2} \mathrm{~d} x \\
& \geq(4+\alpha) B(t) .
\end{aligned}
$$

It follows from the divergence theorem that

$$
\begin{aligned}
B^{\prime}(t) & =\int_{D}\left(f g u_{t}-g g^{\prime}|\nabla u|^{2} u_{t}-g^{2} \nabla u \cdot \nabla u_{t}\right) \mathrm{d} x \\
& =\int_{D}\left(f g u_{t}-g g^{\prime}|\nabla u|^{2} u_{t}\right) \mathrm{d} x+2 \int_{D} g g^{\prime}|\nabla u|^{2} u_{t} \mathrm{~d} x-\int_{D} \nabla\left(g^{2} u_{t}\right) \cdot \nabla u \mathrm{~d} x \\
& =\int_{D} f g u_{t} \mathrm{~d} x+\int_{D} g g^{\prime}|\nabla u|^{2} u_{t} \mathrm{~d} x+\int_{D} g^{2} u_{t} \Delta u \mathrm{~d} x \\
& =\int_{D} g u_{t}\left(f+g^{\prime}|\nabla u|^{2}+g \Delta u\right) \mathrm{d} x \\
& =\int_{D} g k^{\prime}\left(u_{t}\right)^{2} \mathrm{~d} x \geq 0 .
\end{aligned}
$$

Consequently, $B(t)$ is a nondecreasing function in $t$ and

$$
B(t) \geq B(0) \geq 0
$$

By the Schwarz inequality, (2.3), and (2.4), we have

$$
\begin{aligned}
\left(1+\frac{\alpha}{4}\right) A^{\prime} B & \leq \frac{A^{\prime 2}}{4}=\left(\int_{D} g k^{\prime} u u_{t} \mathrm{~d} x\right)^{2} \leq \int_{D} g k^{\prime}\left(u_{t}\right)^{2} \mathrm{~d} x \int_{D} g k^{\prime} u^{2} \mathrm{~d} x \\
& =B^{\prime}(t) \int_{D} g k^{\prime} u^{2} \mathrm{~d} x .
\end{aligned}
$$

It follows from (2.1) and (2.2) that 


$$
\begin{aligned}
A(t) & =\int_{D} G(u) \mathrm{d} x=2 \int_{D}\left(\int_{0}^{u} s g(s) k^{\prime}(s) \mathrm{d} s\right) \mathrm{d} x \\
& =\int_{D}\left(g(u) k^{\prime}(u) u^{2}-\int_{0}^{u}\left(g(s) k^{\prime}(s)\right)^{\prime} s^{2} \mathrm{~d} s\right) \mathrm{d} x \\
& \geq \int_{D} g k^{\prime} u^{2} \mathrm{~d} x .
\end{aligned}
$$

Thus,

$$
\left(1+\frac{\alpha}{4}\right) A^{\prime} B \leq A B^{\prime}
$$

which implies

$$
\frac{\mathrm{d}}{\mathrm{d} t}\left(B A^{-1-\frac{\alpha}{4}}\right) \geq 0
$$

With (2.3) and (2.5), we get

$$
-\frac{4}{\alpha(\alpha+4)}\left(A^{-\frac{\alpha}{4}}\right)^{\prime}=\frac{1}{\alpha+4} A^{\prime} A^{-1-\frac{\alpha}{4}} \geq B A^{-1-\frac{\alpha}{4}} \geq M:=B(0)[A(0)]^{-1-\frac{\alpha}{4}} .
$$

Integrate (2.6) over $[0, t]$ to get

$$
[A(t)]^{-\frac{\alpha}{4}} \leq[A(0)]^{-\frac{\alpha}{4}}-\frac{\alpha(\alpha+4)}{4} M t
$$

which cannot hold for

$$
t \geq T:=\frac{4}{\alpha(\alpha+4)} A(0) B^{-1}(0) .
$$

Hence, $u(x, t)$ must blow up at some finite time $t^{*}<T$. The proof is complete.

\section{Global solution}

In order to get the sufficient conditions for the existence of the global solution and the explicit exponential decay bounds for the solution, we suppose the following:

$$
\begin{aligned}
& s(f(s) g(s))^{\prime}+s^{2} g^{\prime}(s) \geq f(s) g(s), \quad s \in \mathbb{R}^{+}, \\
& \frac{f^{\prime}(s)}{k^{\prime}(s)} \leq p(s), \quad \frac{1}{k^{\prime}(s)}\left(\frac{k^{\prime}(s)}{g(s)}\right)^{\prime} \leq q(s), \quad s \in \overline{\mathbb{R}^{+}},
\end{aligned}
$$

where $p(s)$ and $q(s)$ are nondecreasing functions of $s$. Since the solution of problem (1.1) might blow up in a finite time $t^{*}$, the solution exists in an internal $(0, \gamma)$ with $\gamma<t^{*}$. Further we define

$$
u_{m}:=\max _{D \times(0, \gamma)} u(x, t) \quad(<+\infty)
$$

Next, we give two lemmas from which the main results of this section are derived. 
Lemma 3.1 Let $u$ be a classical solution of the problem (1.1). Suppose that (3.2) holds and

$$
-c \leq \frac{g(h)}{k^{\prime}(h)}[\nabla \cdot(g(h) \nabla h)+f(h)] \leq 0, \quad x \in D,
$$

where $c$ is a positive constant. Then

$$
-c \leq g u_{t} \leq 0, \quad(x, t) \in D \times(0, \gamma) .
$$

Proof Construct an auxiliary function

$$
z(x, t):=g(u) u_{t}
$$

from which we have

$$
\begin{aligned}
& \nabla z=g^{\prime} \nabla u u_{t}+g \nabla u_{t}, \\
& \Delta z=g^{\prime \prime}|\nabla u|^{2} u_{t}+2 g^{\prime} \nabla u_{t} \cdot \nabla u+g^{\prime} \Delta u u_{t}+g \Delta u_{t},
\end{aligned}
$$

and

$$
\begin{aligned}
z_{t}= & g^{\prime}\left(u_{t}\right)^{2}+g u_{t t}=g^{\prime}\left(u_{t}\right)^{2}+g\left(\frac{g}{k^{\prime}} \Delta u+\frac{g^{\prime}}{k^{\prime}}|\nabla u|^{2}+\frac{f}{k^{\prime}}\right)_{t} \\
= & g^{\prime}\left(u_{t}\right)^{2}+\left(\frac{g g^{\prime}}{k^{\prime}}-\frac{g^{2} k^{\prime \prime}}{k^{\prime 2}}\right) \Delta u u_{t}+\frac{g^{2}}{k^{\prime}} \Delta u_{t}+\left(\frac{g g^{\prime \prime}}{k^{\prime}}-\frac{g g^{\prime} k^{\prime \prime}}{k^{\prime 2}}\right)|\nabla u|^{2} u_{t}+2 \frac{g g^{\prime}}{k^{\prime}} \nabla u_{t} \cdot \nabla u \\
& +\left(\frac{f^{\prime} g}{k^{\prime}}-\frac{f g k^{\prime \prime}}{k^{\prime 2}}\right) u_{t} .
\end{aligned}
$$

It follows from (3.5), (3.6), (3.7), and the first equation of (1.1) that

$$
\frac{g}{k^{\prime}} \Delta z-z_{t}+\frac{f^{\prime}}{k^{\prime}} z-\frac{1}{k^{\prime}}\left(\frac{k^{\prime}}{g}\right)^{\prime} z^{2}=0
$$

The comparison principle [15], (3.2), (3.3), and (3.8) imply (3.4) holds. The proof is complete.

In the following, we use the first Dirichlet eigenvalue $\lambda_{1}$ of the Laplacian and the corresponding eigenfunction $\Phi_{1}$ for a region $\tilde{D} \supseteq D$ :

$$
\begin{cases}\Delta \Phi_{1}(x)+\lambda_{1} \Phi_{1}(x)=0, & x \in \tilde{D}, \\ \Phi_{1}(x)=0, & x \in \partial \tilde{D} .\end{cases}
$$

Further since $\Phi_{1}(x)$ is determined up to an arbitrary multiplicative constant, we can normalize $\Phi_{1}(x)$ by

$$
\max _{\tilde{D}} \Phi_{1}(x)=1 .
$$


Lemma 3.2 Let $u$ be a classical solution of the problem (1.1). Suppose that assumptions (3.1), (3.2), and (3.3) hold and

$$
\left(\frac{k^{\prime}(s)}{g(s)}\right)^{\prime} \geq 0, \quad s \in \mathbb{R}^{+}
$$

Then $u(x, t)$ satisfies the following inequality:

$$
0 \leq u(x, t) \leq \Gamma_{1} \exp \left[-\frac{g\left(u_{m}\right)}{k^{\prime}\left(u_{m}\right)}\left(\lambda_{1}-\frac{f\left(u_{m}\right)}{u_{m} g\left(u_{m}\right)}\right) t\right], \quad(x, t) \in D \times(0, \gamma),
$$

where

$$
\Gamma_{1}:=\max _{D} \frac{h(x)}{\Phi_{1}(x)}<+\infty .
$$

Proof Construct the following auxiliary function:

$$
v(x, t):=u(x, t) \exp \left(-\frac{f\left(u_{m}\right)}{u_{m} k^{\prime}\left(u_{m}\right)} t\right)
$$

Here, (3.1) and the fact that $g^{\prime} \leq 0$ imply

$$
\left(\frac{f(s)}{s g(s)}\right)^{\prime} \geq 0, \quad s \in \mathbb{R}^{+}
$$

It follows from Lemma 3.1, (3.11), and (3.14) that

$$
\begin{aligned}
\left(\Delta v-\frac{k^{\prime}\left(u_{m}\right)}{g\left(u_{m}\right)} v_{t}\right) \exp \left(\frac{f\left(u_{m}\right)}{u_{m} k^{\prime}\left(u_{m}\right)} t\right) & =\Delta u-\frac{k^{\prime}\left(u_{m}\right)}{g\left(u_{m}\right)} u_{t}+\frac{f\left(u_{m}\right)}{u_{m} g\left(u_{m}\right)} u \\
& \geq \Delta u-\frac{k^{\prime}(u)}{g(u)} u_{t}+\frac{f(u)}{g(u)}=-\frac{g^{\prime}(u)}{g(u)}|\nabla u|^{2} \geq 0 .
\end{aligned}
$$

Thus, we have

$$
\begin{cases}\Delta v-\frac{k^{\prime}\left(u_{m}\right)}{g\left(u_{m}\right)} v_{t} \geq 0, & (x, t) \in D \times(0, \gamma) \\ v(x, t)=0, & (x, t) \in \partial D \times(0, \gamma) \\ v(x, 0)=h(x), & x \in \bar{D} .\end{cases}
$$

Let

$$
w(x, t):=\Gamma_{1} \Phi_{1}(x) \exp \left(-\frac{\lambda_{1} g\left(u_{m}\right)}{k^{\prime}\left(u_{m}\right)} t\right) .
$$

With (3.9), (3.13), and (3.16), we have

$$
\begin{cases}\Delta w-\frac{k^{\prime}\left(u_{m}\right)}{g\left(u_{m}\right)} w_{t}=0, & (x, t) \in D \times(0, \gamma), \\ w(x, t) \geq 0, & (x, t) \in \partial D \times(0, \gamma), \\ w(x, 0)=\Gamma_{1} \Phi_{1}(x) \geq h(x), & x \in \bar{D} .\end{cases}
$$


It follows from (3.15), (3.17), and the comparison principle that

$$
v(x, t) \leq w(x, t)
$$

which implies

$$
u(x, t) \leq \Gamma_{1} \Phi_{1}(x) \exp \left[-\frac{g\left(u_{m}\right)}{k^{\prime}\left(u_{m}\right)}\left(\lambda_{1}-\frac{f\left(u_{m}\right)}{u_{m} g\left(u_{m}\right)}\right) t\right] .
$$

With (3.10) and (3.18), we derive (3.12). The proof is complete.

Next, we can get Theorem 3.1 from Lemmas 3.1-3.2.

Theorem 3.1 Let $u$ be a classical solution of the problem (1.1). Suppose that (3.1), (3.2), (3.3), and (3.11) hold and

$$
\frac{f\left(\Gamma_{1}\right)}{\Gamma_{1} g\left(\Gamma_{1}\right)}<\lambda_{1}
$$

Then we have

$$
t^{*}=\infty
$$

and

$$
\max _{D} \frac{f(u(x, t))}{u(x, t) g(u(x, t))}<\lambda_{1}, \quad t \in[0, \infty)
$$

Proof We assume that (3.20) cannot hold. There exists a first time $\tilde{t}<\infty$ for which $\frac{f(u)}{u g(u)}$ reaches the value $\lambda_{1}$. Thus, we have

$$
\max _{D} \frac{f(u(x, \tilde{t}))}{u(x, \tilde{t}) g(u(x, \tilde{t}))}=\lambda_{1}
$$

The fact that $\frac{f(s)}{s g(s)}$ is a nondecreasing functions in $s$, (3.19), and Lemma 3.2 imply

$$
u(x, t) \leq \Gamma_{1}, \quad(x, t) \in D \times[0, \tilde{t}]
$$

and

$$
\frac{f(u(x, t))}{u(x, t) g(u(x, t))} \leq \frac{f\left(\Gamma_{1}\right)}{\Gamma_{1} g\left(\Gamma_{1}\right)}<\lambda_{1}, \quad(x, t) \in D \times[0, \tilde{t}] .
$$

Hence, we have

$$
\max _{D} \frac{f(u(x, \tilde{t}))}{u(x, \tilde{t}) g(u(x, \tilde{t}))}<\lambda_{1}
$$

which contradicts with the inequality (3.21). So we conclude that $\tilde{t}=\infty$ and (3.20) holds. The proof is complete. 


\section{Exponential decay estimate}

In this section, we will use a comma to denote partial differentiation and adopt the summation convection, i.e., if an index is repeated, summation from 1 to $N$ is understood, for example,

$$
u_{, i} u_{, k} u_{, i k}=\sum_{i, k=1}^{N} \frac{\partial u}{\partial x_{i}} \frac{\partial u}{\partial x_{k}} \frac{\partial^{2} u}{\partial x_{i} \partial x_{k}} .
$$

Hence, the differentiated form of the first equation of (1.1) is

$$
k^{\prime}(u) u_{t}=\left(g(u) u_{, i}\right)_{, i}+f(u)
$$

In order to get the exponential decay bounds for the derivatives of the solution, we consider

$$
\Psi(x, t):=\left(g^{2}(u)|\nabla u|^{2}+2 \int_{0}^{u} f(s) g(s) \mathrm{d} s+2 a \int_{0}^{u} s g(s) \mathrm{d} s\right) e^{2 \beta t},
$$

where $a \geq 1$ and $0<\beta \leq 1$ are some positive constants to be determined. Our main result is Theorem 4.1.

Theorem 4.1 Let $u$ be the classical solution of the problem (1.1). Suppose the following.

(i) The inequalities (3.1), (3.2), (3.3), and (3.11) hold and

$$
0<k^{\prime}(s) \leq b \leq 1, \quad s \in \mathbb{R}^{+},
$$

where $b$ is a positive constant.

(ii)

$$
\lim _{s \rightarrow 0^{+}} s g(s)=0 .
$$

(iii)

$$
\frac{a}{b}:=M+\beta<\frac{\pi^{2}}{4 d^{2}} g\left(\Gamma_{1}\right)-\frac{f\left(\Gamma_{1}\right)}{\Gamma_{1}},
$$

where $d$ is the in-radius of $D$ and

$$
M:=c \max _{s \in\left[0, \Gamma_{1}\right]}\left\{\frac{1}{k^{\prime}(s)}\left(\frac{k^{\prime}(s)}{g(s)}\right)^{\prime}\right\}
$$

with $c$ given in Lemma 3.2. Thus, $\Psi(x, t)$ takes its maximum value at $t=0$, i.e.,

$$
g^{2}(u)|\nabla u|^{2}+2 \int_{0}^{u} f(s) g(s) \mathrm{d} s+2 a \int_{0}^{u} s g(s) \mathrm{d} s \leq H^{2} e^{-2 \beta t}, \quad(x, t) \in D \times(0, \infty),
$$

with

$$
H^{2}:=\max _{D}\left\{g^{2}(h)|\nabla h|^{2}+2 \int_{0}^{h} f(s) g(s) \mathrm{d} s+2 a \int_{0}^{h} s g(s) \mathrm{d} s\right\} .
$$


Proof The theorem will be proved in three steps.

Step 1. Differentiating (4.2), we get

$$
\begin{aligned}
& \Psi_{, k}=2\left(g g^{\prime}|\nabla u|^{2} u_{, k}+g^{2} u_{, i} u_{, i k}+f g u_{, k}+a u g u_{, k}\right) \mathrm{e}^{2 \beta t}, \\
&\left(g \Psi_{, k}\right)_{, k}=\left[2\left(g^{2} g^{\prime}|\nabla u|^{2} u_{, k}+g^{3} u_{, i} u_{, i k}+f g^{2} u_{, k}+a u g^{2} u_{, k}\right) \mathrm{e}^{2 \beta t}\right]_{, k} \\
&=2 \mathrm{e}^{2 \beta t}\left(2 g\left(g^{\prime}\right)^{2}|\nabla u|^{4}+g^{2} g^{\prime \prime}|\nabla u|^{4}+4 g^{2} g^{\prime} u_{, i} u_{, k} u_{, i k}\right. \\
&+g^{2} g^{\prime}|\nabla u|^{2} \Delta u+g^{2}\left(g u_{, i k}\right)_{, k} u_{, i} \\
&+g^{3} u_{, i k} u, i k+f^{\prime} g^{2}|\nabla u|^{2}+2 f g g^{\prime}|\nabla u|^{2}+f g^{2} \Delta u+a g^{2}|\nabla u|^{2} \\
&+\left.2 a u g g^{\prime}|\nabla u|^{2}+a u g^{2} \Delta u\right),
\end{aligned}
$$

and

$$
\begin{aligned}
\Psi_{t}= & 2 \mathrm{e}^{2 \beta t}\left(g g^{\prime}|\nabla u|^{2} u_{t}+g^{2} u_{, i} u_{t, i}+f g u_{t}+a u g u_{t}+\beta g^{2}|\nabla u|^{2}+2 \beta \int_{0}^{u} f(s) g(s) \mathrm{d} s\right. \\
& \left.+2 a \beta \int_{0}^{u} s g(s) \mathrm{d} s\right) .
\end{aligned}
$$

It follows from the first equation of (1.1) that

$$
\Delta u=-\frac{g^{\prime}}{g}|\nabla u|^{2}-\frac{f}{g}+\frac{k^{\prime}}{g} u_{t} .
$$

Next, substituting (4.9) into (4.7), we have

$$
\begin{aligned}
\left(g \Psi_{, k}\right)_{, k}= & 2 \mathrm{e}^{2 \beta t}\left(g\left(g^{\prime}\right)^{2}|\nabla u|^{4}+g^{2} g^{\prime \prime}|\nabla u|^{4}+4 g^{2} g^{\prime} u_{, i} u_{, k} u_{, i k}+g g^{\prime} k^{\prime}|\nabla u|^{2} u_{t}\right. \\
& +g^{2}(g u, i k)_{, k} u_{, i}+g^{3} u_{, i k} u_{, i k}+f^{\prime} g^{2}|\nabla u|^{2}+f g k^{\prime} u_{t}-f^{2} g+a g^{2}|\nabla u|^{2} \\
& \left.+a u g g^{\prime}|\nabla u|^{2}+a u g k^{\prime} u_{t}-a u f g\right) .
\end{aligned}
$$

Differentiating (4.1), we have

$$
\left(g u_{, k}\right)_{, k i}=\left(k^{\prime} u_{t}-f\right)_{, i}
$$

i.e.,

$$
\left(g^{\prime} u_{, i} u_{, k}+g u_{, i k}\right)_{, k}=k^{\prime \prime} u_{, i} u_{t}+k^{\prime} u_{t, i}-f^{\prime} u_{, i} .
$$

It follows from (4.11) that

$$
\begin{aligned}
\left(g u_{, i k}\right)_{, k} & =k^{\prime \prime} u_{, i} u_{t}+k^{\prime} u_{t, i}-f^{\prime} u_{, i}-\left(g^{\prime} u_{, i} u_{, k}\right)_{, k} \\
& =k^{\prime \prime} u_{, i} u_{t}+k^{\prime} u_{t, i}-f^{\prime} u_{, i}-g^{\prime \prime}|\nabla u|^{2} u_{, i}-g^{\prime} u_{, k} u_{, i k}-g^{\prime} u_{, i} \Delta u
\end{aligned}
$$

Multiplied by $g^{2} u_{, i}$ from (4.12), we have

$$
\begin{aligned}
g^{2}\left(g u_{, i k}\right)_{, k} u_{, i}= & g^{2} k^{\prime \prime}|\nabla u|^{2} u_{t}+g^{2} k^{\prime} u_{, i} u_{t, i}-f^{\prime} g^{2}|\nabla u|^{2} \\
& -g^{2} g^{\prime \prime}|\nabla u|^{4}-g^{2} g^{\prime} u_{, i} u_{, k} u_{, i k}-g^{2} g^{\prime}|\nabla u|^{2} \Delta u .
\end{aligned}
$$


Substituting (4.9) into (4.13), we get

$$
\begin{aligned}
g^{2}\left(g u_{, i k}\right)_{, k} u_{, i}= & g^{2} k^{\prime \prime}|\nabla u|^{2} u_{t}+g^{2} k^{\prime} u_{, i} u_{t, i}-f^{\prime} g^{2}|\nabla u|^{2}-g^{2} g^{\prime \prime}|\nabla u|^{4}-g^{2} g^{\prime} u_{, i} u_{, k} u_{, i k} \\
& -g g^{\prime} k^{\prime}|\nabla u|^{2} u_{t}+f g g^{\prime}|\nabla u|^{2}+g\left(g^{\prime}\right)^{2}|\nabla u|^{4} .
\end{aligned}
$$

We substitute (4.14) into (4.10) to obtain

$$
\begin{aligned}
\left(g \Psi_{, k}\right)_{, k}= & 2 \mathrm{e}^{2 \beta t}\left(2 g\left(g^{\prime}\right)^{2}|\nabla u|^{4}+3 g^{2} g^{\prime} u_{, i} u_{, k} u_{, i k}+g^{2} k^{\prime \prime}|\nabla u|^{2} u_{t}\right. \\
& +g^{2} k^{\prime} u_{, i} u_{t, i}+f g g^{\prime}|\nabla u|^{2}+g^{3} u_{, i k} u_{, i k}+f g k^{\prime} u_{t} \\
& \left.-f^{2} g+a g^{2}|\nabla u|^{2}+a u g g^{\prime}|\nabla u|^{2}+a u g k^{\prime} u_{t}-a u f g\right) .
\end{aligned}
$$

It follows from (4.6) that

$$
g^{2} u_{, i} u_{, i k}=\frac{1}{2} \mathrm{e}^{-2 \beta t} \Psi_{, k}-g g^{\prime}|\nabla u|^{2} u_{, k}-f g u_{, k}-a u g u_{, k} .
$$

Substituting (4.16) into (4.15), we get

$$
\begin{aligned}
\left(g \Psi_{, k}\right)_{, k}= & 2 \mathrm{e}^{2 \beta t}\left(-g\left(g^{\prime}\right)^{2}|\nabla u|^{4}+\frac{3}{2} \mathrm{e}^{-2 \beta t} g^{\prime} u_{, k} \Psi_{, k}-2 f g g^{\prime}|\nabla u|^{2}\right. \\
& -2 a u g g^{\prime}|\nabla u|^{2}+g^{2} k^{\prime \prime}|\nabla u|^{2} u_{t}+g^{2} k^{\prime} u_{, i} u_{t, i}+g^{3} u_{, i k} u_{, i k}+f g k^{\prime} u_{t} \\
& \left.-f^{2} g+a g^{2}|\nabla u|^{2}+a u g k^{\prime} u_{t}-a u f g\right) .
\end{aligned}
$$

It follows from (4.8) and (4.17) that

$$
\begin{aligned}
\left(g \Psi_{, k}\right)_{, k}-k^{\prime} \Psi_{t}= & 2 \mathrm{e}^{2 \beta t}\left(-g\left(g^{\prime}\right)^{2}|\nabla u|^{4}+\frac{3}{2} \mathrm{e}^{-2 \beta t} g^{\prime} u_{, k} \Psi_{, k}-2 f g g^{\prime}|\nabla u|^{2}-2 a u g g^{\prime}|\nabla u|^{2}\right. \\
& +g^{2} k^{\prime \prime}|\nabla u|^{2} u_{t}+g^{3} u_{, i k} u_{, i k}-f^{2} g+a g^{2}|\nabla u|^{2}-a u f g-g g^{\prime} k^{\prime}|\nabla u|^{2} u_{t} \\
& \left.-\beta g^{2} k^{\prime}|\nabla u|^{2}-2 \beta k^{\prime} \int_{0}^{u} f(s) g(s) \mathrm{d} s-2 a \beta k^{\prime} \int_{0}^{u} s g(s) \mathrm{d} s\right) .
\end{aligned}
$$

Next, we use the Cauchy-Schwarz inequality in the following form:

$$
|\nabla u|^{2} u_{, i k} u_{, i k} \geq u_{, k} u_{, i k} u_{, j} u_{, i j}
$$

It follows from (4.6) that

$$
u_{, k} u_{, i k}=u_{, j} u_{, i j}=\frac{1}{g^{2}}\left(\frac{1}{2} \mathrm{e}^{-2 \beta t} \Psi_{, i}-g g^{\prime}|\nabla u|^{2} u_{, i}-f g u_{, i}-a u g u_{, i}\right) .
$$

Further, with (4.19) and (4.20), we obtain

$$
\begin{aligned}
u_{, i k} u_{, i k} & \geq \frac{1}{g^{4}|\nabla u|^{2}}\left(\frac{1}{2} \mathrm{e}^{-2 \beta t} \Psi_{, i}-g g^{\prime}|\nabla u|^{2} u_{, i}-f g u_{, i}-a u g u_{, i}\right)^{2} \\
& =\frac{1}{4 g^{4}|\nabla u|^{2}} \mathrm{e}^{-4 \beta t}\left(\Psi_{, i}\right)^{2}-\frac{1}{g^{3}|\nabla u|^{2}}\left(g^{\prime}|\nabla u|^{2} u_{, i}+a u u_{, i}+f u_{, i}\right) \mathrm{e}^{-2 \beta t} \Psi_{, i}
\end{aligned}
$$




$$
\begin{aligned}
& +\frac{1}{g^{2}}\left(\left(g^{\prime}\right)^{2}|\nabla u|^{4}+a^{2} u^{2}+f^{2}+2 a u g^{\prime}|\nabla u|^{2}+2 a u f+2 f g^{\prime}|\nabla u|^{2}\right), \\
& x \in D \backslash W
\end{aligned}
$$

where $W:=\{x \in D: \nabla u(x, t)=0\}$ is the set of critical points of $u$. Substituting (4.21) into (4.18), we have

$$
\begin{aligned}
\frac{g}{k^{\prime}} \Delta \Psi & +\frac{1}{k^{\prime}}\left\{\left(g^{\prime}-\frac{\mathrm{e}^{-2 \beta t}}{2 g|\nabla u|^{2}}\right) \nabla \Psi-\left[g^{\prime}-\frac{2}{|\nabla u|^{2}}(f+a u)\right] \nabla u\right\} \cdot \nabla \Psi-\Psi_{t} \\
\geq & 2 \mathrm{e}^{2 \beta t}\left\{a \frac{f g u}{k^{\prime}}-2 \beta \int_{0}^{u} f(s) g(s) \mathrm{d} s+a^{2} \frac{g u^{2}}{k^{\prime}}-2 a \beta \int_{0}^{u} s g(s) \mathrm{d} s\right. \\
& \left.+g^{2}|\nabla u|^{2}\left[\frac{a}{k^{\prime}}-\beta+\frac{g}{k^{\prime}}\left(\frac{k^{\prime}}{g}\right)^{\prime} u_{t}\right]\right\} .
\end{aligned}
$$

Integrating (3.1) from 0 to $u(x, t)$ and using (4.4), we get

$$
f g u-2 \int_{0}^{u} f(s) g(s) \mathrm{d} s+g u^{2}-2 \int_{0}^{u} s g(s) \mathrm{d} s \geq 0 .
$$

Making use of the fact that $a \geq 1,(4.3)$, and (4.23), we have

$$
\begin{aligned}
& a \frac{f g u}{k^{\prime}}-2 \beta \int_{0}^{u} f(s) g(s) \mathrm{d} s+a^{2} \frac{g u^{2}}{k^{\prime}}-2 a \beta \int_{0}^{u} s g(s) \mathrm{d} s \\
& \quad \geq a \frac{f g u}{b}-2 a \beta \int_{0}^{u} f(s) g(s) \mathrm{d} s+a^{2} \frac{g u^{2}}{b}-2 a \beta \int_{0}^{u} s g(s) \mathrm{d} s \\
& \geq a f g u-2 a \beta \int_{0}^{u} f(s) g(s) \mathrm{d} s+a^{2} g u^{2}-2 a \beta \int_{0}^{u} s g(s) \mathrm{d} s \\
& \geq a\left(f g u-2 \int_{0}^{u} f(s) g(s) \mathrm{d} s+a g u^{2}-2 \int_{0}^{u} s g(s) \mathrm{d} s\right) \\
& \geq f g u-2 \int_{0}^{u} f(s) g(s) \mathrm{d} s+g u^{2}-2 \int_{0}^{u} s g(s) \mathrm{d} s \geq 0 .
\end{aligned}
$$

Moreover, by (4.5), it is easy to see

$$
\frac{f\left(\Gamma_{1}\right)}{\Gamma_{1}}<\frac{\pi^{2}}{4 d^{2}} g\left(\Gamma_{1}\right) \leq \lambda_{1} g\left(\Gamma_{1}\right)
$$

It follows from Theorem 3.1 that

$$
\frac{f\left(u_{m}\right)}{u_{m} g\left(u_{m}\right)}<\lambda_{1}
$$

which implies

$$
u_{m} \leq \Gamma_{1} .
$$

Next, it follows from (4.25), (4.3), (4.5), and Lemma 3.1 that

$$
\frac{a}{k^{\prime}}-\beta+\frac{g}{k^{\prime}}\left(\frac{k}{g}\right)^{\prime} u_{t} \geq \frac{a}{b}-\beta-\frac{c}{k^{\prime}}\left(\frac{k}{g}\right)^{\prime} \geq \frac{a}{b}-\beta-M=0 .
$$


Consequently, (4.22), (4.24), and (4.26) imply

$$
\begin{aligned}
& \frac{g}{k^{\prime}} \Delta \Psi+\frac{1}{k^{\prime}}\left\{\left(g^{\prime}-\frac{\mathrm{e}^{-2 \beta t}}{2 g|\nabla u|^{2}}\right) \nabla \Psi-\left[g^{\prime}-\frac{2}{|\nabla u|^{2}}(f+a u)\right] \nabla u\right\} \cdot \nabla \Psi-\Psi_{t} \geq 0, \\
& \quad x \in D \backslash W .
\end{aligned}
$$

By means of the maximum principle, we have the following possible cases where $\Psi$ may take its maximum value:

(a) on the boundary $\partial D \times(0, T)$,

(b) at a point where $\nabla u=0$,

(c) for $t=0$.

Step 2. We first exclude the case (a). Assume $\Psi(x, t)$ takes its maximum value at $\hat{Q}=(\hat{x}, \hat{t})$ on $\partial D$. Since $u=0$ on $\partial D$, we have

$$
\frac{\partial \Psi}{\partial n}=2\left[g g^{\prime}\left(\frac{\partial u}{\partial n}\right)^{3}+g^{2} \frac{\partial^{2} u}{\partial n^{2}} \frac{\partial u}{\partial n}\right] \mathrm{e}^{2 \beta t} .
$$

With (1.1) and $f(0)=0$, evaluated on $\partial D \in C^{2, \varepsilon}$, we get

$$
g^{\prime}\left(\frac{\partial u}{\partial n}\right)^{2}+g\left[\frac{\partial^{2} u}{\partial n^{2}}+(N-1) K \frac{\partial u}{\partial n}\right]=0,
$$

where $K$ is the average curvature of $\partial D$. By (4.27) and (4.28), we are led to

$$
\frac{\partial \Psi}{\partial n}=\left[-2(N-1) k g^{2}\left(\frac{\partial u}{\partial n}\right)^{2}\right] e^{2 \beta t} \leq 0, \quad x \in \partial D .
$$

Hence, we have

$$
\left.\frac{\partial \Psi}{\partial n}\right|_{\hat{Q}=(\hat{x}, \hat{t})} \leq 0,
$$

which contradicts with the maximum principle. Hence, $\Psi$ cannot take its maximum value on $\partial D$.

Step 3 . In the following, we exclude the case (b). Assume $\Psi(x, t)$ takes its maximum value at a critical point $\bar{Q}=(\bar{x}, \bar{t})$.

Thus we have

$$
\Psi(x, t) \leq \Psi(\bar{x}, \bar{t}), \quad(x, t) \in D \times(0,+\infty) .
$$

Replacing $t$ with $\bar{t}$ in (4.29), we obtain

$$
g^{2}(u(x, \bar{t}))|\nabla u(x, \bar{t})|^{2} \leq 2 \int_{u(x, \bar{t})}^{u(\bar{x}, \bar{t})} f(s) g(s) \mathrm{d} s+2 a \int_{u(x, \bar{t})}^{u(\bar{x}, \bar{t})} s g(s) \mathrm{d} s,
$$

from which we have

$$
g^{2}(u(x, \bar{t}))|\nabla u(x, \bar{t})|^{2} \leq 2 \int_{u(x, \bar{t})}^{u_{M}} f(s) g(s) \mathrm{d} s+2 a \int_{u(x, \bar{t})}^{u_{M}} s g(s) \mathrm{d} s, \quad x \in D,
$$

where $u_{M}=\max _{D} u(x, \bar{t})$. 
Here, (3.1) and the fact that $g^{\prime}(s) \leq 0$ imply

$$
\left(\frac{f(s) g(s)}{s}\right)^{\prime} \geq 0, \quad s \in \mathbb{R}^{+}
$$

Next, making use of Cauchy's mean value theorem and of (4.31), we get

$$
\begin{aligned}
2 \int_{u(x, \bar{t})}^{u_{M}} f(s) g(s) \mathrm{d} s & =\frac{f(\xi) g(\xi)}{\xi}\left(u_{M}^{2}-u^{2}(x, \bar{t})\right) \leq \frac{f\left(u_{M}\right) g\left(u_{M}\right)}{u_{M}}\left(u_{M}^{2}-u^{2}(x, \bar{t})\right) \\
& \leq \frac{f\left(u_{M}\right) g(u(x, \bar{t}))}{u_{M}}\left(u_{M}^{2}-u^{2}(x, \bar{t})\right),
\end{aligned}
$$

where $\xi$ is some intermediate value between $u(x, \bar{t})$ and $u_{M}$. The fact that $g^{\prime}(s) \leq 0$ implies

$$
2 \int_{u(x, \bar{t})}^{u_{M}} \operatorname{sg}(s) \mathrm{d} s \leq g(u(x, \bar{t}))\left(u_{M}^{2}-u^{2}(x, \bar{t})\right)
$$

Hence, inserting (4.32) and (4.33) in (4.30), we get

$$
|\nabla u(x, \bar{t})|^{2} \leq\left(\frac{f\left(u_{M}\right)}{u_{M}}+a\right)\left(\frac{u_{M}^{2}-u^{2}(x, \bar{t})}{g\left(u_{M}\right)}\right), \quad x \in D
$$

With (4.34), we have

$$
\frac{d u}{\sqrt{u_{M}^{2}-u^{2}(x, \bar{t})}} \leq \sqrt{\left(\frac{f\left(u_{M}\right)}{u_{M}}+a\right) \frac{1}{g\left(u_{M}\right)}} d \tau
$$

Integrate (4.35) on a straight line from $\bar{x}$ to the nearest point $x_{0} \in \partial D$ to obtain

$$
\frac{\pi}{2} \leq \sqrt{\left(\frac{f\left(u_{M}\right)}{u_{M}}+a\right) \frac{1}{g\left(u_{M}\right)}}\left|\bar{x} x_{0}\right| \leq \sqrt{\left(\frac{f\left(u_{M}\right)}{u_{M}}+a\right) \frac{1}{g\left(u_{M}\right)}} d,
$$

from which we have

$$
\left(\frac{f\left(u_{M}\right)}{u_{M}}+a\right) \frac{1}{g\left(u_{M}\right)} \geq \frac{\pi^{2}}{4 d^{2}}
$$

We note that (3.1) and the fact that $g^{\prime}(s) \leq 0$ ensure $\frac{f(s)}{s}$ is a nondecreasing function. It follows from (4.25) and (4.5) that

$$
\frac{f\left(u_{m}\right)}{u_{m}} \leq \frac{f\left(\Gamma_{1}\right)}{\Gamma_{1}}<\frac{\pi^{2}}{4 d^{2}} g\left(\Gamma_{1}\right)-\frac{a}{b} \leq \frac{\pi^{2}}{4 d^{2}} g\left(u_{m}\right)-\frac{a}{b} \leq \frac{\pi^{2}}{4 d^{2}} g\left(u_{m}\right)-a,
$$

which with $u_{M} \leq u_{m}$ implies

$$
\left(\frac{f\left(u_{M}\right)}{u_{M}}+a\right) \frac{1}{g\left(u_{M}\right)} \leq\left(\frac{f\left(u_{m}\right)}{u_{m}}+a\right) \frac{1}{g\left(u_{m}\right)}<\frac{\pi^{2}}{4 d^{2}}
$$

which contradicts with (4.36). The proof is complete. 


\section{Applications}

When $k(u) \equiv u$ and $g(u) \equiv 1$ or $k(u) \equiv u$, the conclusions of Theorems 2.1, 3.1 and 4.1 still hold true. In this sense, our results extend and supplement those of [16, 17]

In what follows, as applications of the obtained results, two examples are presented.

Example 5.1 Let $u$ be a classical solution of the following problem:

$$
\begin{cases}u_{t}=\sqrt{1+\frac{1}{u}} \Delta u-\frac{1}{2 u} \sqrt{1+\frac{1}{u}}|\nabla u|^{2}+u^{2} \sqrt{u(u+1)}, & (x, t) \in D \times(0, T), \\ u=0, & (x, t) \in \partial D \times(0, T), \\ u(x, 0)=\left(16-|x|^{2}\right)^{2}, & x \in \bar{D},\end{cases}
$$

where $D=\left\{x=\left(x_{1}, x_{2}, x_{3}\right)|| x \mid=\left(\sum_{i=1}^{3} x_{i}^{2}\right)^{1 / 2}<4\right\}$ is the ball of $\mathbb{R}^{3}$. The above problem can be transformed into the following problem:

$$
\begin{cases}(2 \sqrt{u+1})_{t}=\nabla \cdot\left(\frac{1}{\sqrt{u}} \nabla u\right)+u^{\frac{5}{2}}, & (x, t) \in D \times(0, T), \\ u=0, & (x, t) \in \partial D \times(0, T), \\ u(x, 0)=\left(16-|x|^{2}\right)^{2}, & x \in \bar{D} .\end{cases}
$$

Now,

$$
k(u)=2 \sqrt{u+1}, \quad g(u)=\frac{1}{\sqrt{u}}, \quad f(u)=u^{\frac{5}{2}}, \quad h(x)=\left(16-|x|^{2}\right)^{2} .
$$

We have

$$
\begin{aligned}
& F(u)=\int_{0}^{u} f(s) g(s) \mathrm{d} s=\int_{0}^{u} s^{2} \mathrm{~d} s=\frac{u^{3}}{3}, \\
& G(u)=2 \int_{0}^{u} s k^{\prime}(s) g(s) \mathrm{d} s=2 \int_{0}^{u} \sqrt{\frac{s}{s+1}} \mathrm{~d} s=2 \ln (\sqrt{u+1}-\sqrt{u})+2 \sqrt{u(u+1)} .
\end{aligned}
$$

By choosing $\alpha=1$, it is easy to check that (2.1) and (2.2) hold with

$$
\begin{aligned}
A(0) & =\int_{D} G(h(x)) \mathrm{d} x=2 \int_{D} \ln (\sqrt{h+1}-\sqrt{h})+\sqrt{h(h+1)} \mathrm{d} x \\
& =2 \int_{D} \ln \left(\sqrt{\left(16-|x|^{2}\right)^{2}+1}-16+|x|^{2}\right)+\left(16-|x|^{2}\right) \sqrt{\left(16-|x|^{2}\right)^{2}+1} \mathrm{~d} x \\
& =8 \pi \int_{0}^{4}\left[\ln \left(\sqrt{\left(16-r^{2}\right)^{2}+1}-16+r^{2}\right)+\left(16-r^{2}\right) \sqrt{\left(16-r^{2}\right)^{2}+1}\right] r^{2} \mathrm{~d} r \\
& =3.040559 \times 10^{4}
\end{aligned}
$$

and

$$
\begin{aligned}
B(0) & =\int_{D}\left(F(h)-\frac{1}{2} g^{2}(h)|\nabla h|^{2}\right) \mathrm{d} x=\int_{D}\left(\frac{1}{3} h^{3}-\frac{|\nabla h|^{2}}{2 h}\right) \mathrm{d} x \\
& =\int_{D}\left(\frac{1}{3}\left(16-|x|^{2}\right)^{6}-8|x|^{2}\right) \mathrm{d} x=4 \pi \int_{0}^{4}\left(\frac{1}{3}\left(16-r^{2}\right)^{6}-8 r^{2}\right) r^{2} \mathrm{~d} r \\
& =1.022244 \times 10^{8} .
\end{aligned}
$$


It follows from Theorem 2.1 that $u$ blows up in a finite time $t^{*}$ and

$$
t^{*}<T=\frac{4 A(0)}{\alpha(\alpha+4) B(0)}=2.38 \times 10^{-4}
$$

Example 5.2 Let $u$ be a classical solution of the following problem:

$$
\begin{cases}u_{t}=\Delta u-\frac{1}{u+1}|\nabla u|^{2}+u^{2}(u+1), & (x, t) \in D \times(0, T) \\ u=0, & (x, t) \in \partial D \times(0, T) \\ u(x, 0)=\Phi_{1}(x)=\frac{\sin (8|x|)}{8|x|}, & x \in \bar{D},\end{cases}
$$

where $D=\left\{x=\left(x_{1}, x_{2}, x_{3}\right)|| x \mid=\left(\sum_{i=1}^{3} x_{i}^{2}\right)^{1 / 2}<\frac{\pi}{8}\right\}$ is the ball of $\mathbb{R}^{3}, \Phi_{1}(x)$ is the first eigenfunction of $\tilde{D}=D$ and $\max _{D} \Phi_{1}(x)=1$. The above problem may be turned into the following problem:

$$
\begin{cases}(\ln (u+1))_{t}=\nabla \cdot\left(\frac{1}{u+1} \nabla u\right)+u^{2}, & (x, t) \in D \times(0, T), \\ u=0, & (x, t) \in \partial D \times(0, T), \\ u(x, 0)=\Phi_{1}(x)=\frac{\sin (8|x|)}{8|x|}, & x \in \bar{D} .\end{cases}
$$

Now we have

$$
k(u)=\ln (u+1), \quad g(u)=\frac{1}{u+1}, \quad f(u)=u^{2}, \quad h(x)=\Phi_{1}(x)=\frac{\sin (8|x|)}{8|x|} .
$$

Here,

$$
\Gamma_{1}=1, \quad \frac{f\left(\Gamma_{1}\right)}{\Gamma_{1} g\left(\Gamma_{1}\right)}=2, \quad \lambda_{1}=\frac{\pi^{2}}{R^{2}}=64
$$

By choosing $c=31$, it is easy to check that (3.1), (3.2), (3.3), (3.11), and (3.19) hold. It follows from Lemma 3.2 and Theorem 3.1 that $u(x, t)$ is a global solution and

$$
\begin{aligned}
u(x, t) & \leq \Gamma_{1} \exp \left[-\frac{g\left(u_{m}\right)}{k^{\prime}\left(u_{m}\right)}\left(\lambda_{1}-\frac{f\left(u_{m}\right)}{g\left(u_{m}\right) u_{m}}\right) t\right]=\exp \left[-\frac{g\left(u_{m}\right)}{k^{\prime}\left(u_{m}\right)}\left(64-\frac{f\left(u_{m}\right)}{g\left(u_{m}\right) u_{m}}\right) t\right] \\
& \leq \exp \left[-\frac{g\left(\Gamma_{1}\right)}{k^{\prime}\left(\Gamma_{1}\right)}\left(64-\frac{f\left(\Gamma_{1}\right)}{\Gamma_{1} g\left(\Gamma_{1}\right)}\right) t\right]=\mathrm{e}^{-62 t},
\end{aligned}
$$

which is the exponential decay estimate of the solution. By taking $a=b=\beta=1$, it is also easy to check that (4.3), (4.4), and (4.5) hold. It follows from Theorem 4.1 that

$$
g^{2}(u)|\nabla u|^{2}+2 \int_{0}^{u} f(s) g(s) \mathrm{d} s+2 a \int_{0}^{u} s g(s) \mathrm{d} s \leq H^{2} e^{-2 \beta t}, \quad(x, t) \in D \times(0, \infty),
$$

with

$$
\begin{aligned}
H^{2} & =\max _{D}\left\{g^{2}(h)|\nabla h|^{2}+2 \int_{0}^{h} f(s) g(s) \mathrm{d} s+2 a \int_{0}^{h} s g(s) \mathrm{d} s\right\}=\max _{D}\left\{\frac{|\nabla h|^{2}}{(h+1)^{2}}+h^{2}\right\} \\
& =\max _{D}\left\{\frac{\left(\frac{\cos 8|x|}{|x|}-\frac{\sin 8|x|}{8|x|^{2}}\right)^{2}}{\left(\frac{\sin (8|x|)}{8|x|}+1\right)^{2}}+\left(\frac{\sin (8|x|)}{8|x|}\right)^{2}\right\}=7.4 .
\end{aligned}
$$


Hence, we have

$$
|\nabla u|^{2} \leq \frac{1}{g^{2}(u)} H^{2} \mathrm{e}^{-2 \beta t} \leq \frac{1}{g^{2}\left(\Gamma_{1}\right)} H^{2} \mathrm{e}^{-2 \beta t}=29.6 \mathrm{e}^{-2 t},
$$

which is the exponential decay estimate of the gradient for the solution.

\section{Competing interests}

The authors declare that they have no competing interests.

\section{Authors' contributions}

All authors contributed equally to the writing of this paper. All authors read and approved the final manuscript.

\section{Acknowledgements}

This work was supported by the National Natural Science Foundation of China (Nos. 61473180 and 61174082).

Received: 6 March 2015 Accepted: 1 September 2015 Published online: 11 September 2015

\section{References}

1. Levine, HA: The role of critical exponents in blow-up theorems. SIAM Rev. 32, 262-288 (1990)

2. Bandle, C, Brunner, H: Blow-up in diffusion equations: a survey. J. Comput. Appl. Math. 97, 3-22 (1998)

3. Deng, K, Levine, HA: The role of critical exponents in blow-up theorems: the sequel. J. Math. Anal. Appl. 243, 85-126 (2000)

4. Galaktionov, VA, Vázquez, JL: The problem of blow-up in nonlinear parabolic equations. Discrete Contin. Dyn. Syst. 8, 399-433 (2002)

5. Quittner, P, Souplet, P: Superlinear parabolic problems. In: Blow-up, Global Existence and Steady States. Birkhäuser Advanced Texts. Birkhäuser, Basel (2007)

6. Zhang, HL: Blow-up solutions and global solutions for nonlinear parabolic problems. Nonlinear Anal. TMA 69 4567-4574 (2008)

7. Zhang, LL, Zhang, N, Li, LX: Blow-up solutions and global existence for a kind of quasilinear reaction-diffusion equations. Z. Anal. Anwend. 33, 247-258 (2014)

8. Payne, LE, Philippin, GA, Vernier-Piro, S: Blow-up, decay bounds and continuous dependence inequalities for a class of quasilinear parabolic problems. Math. Methods Appl. Sci. 29, 281-295 (2006)

9. Payne, LE, Philippin, GA: Decay bounds for solutions of second order parabolic problems and their derivatives II. Math. Inequal. Appl. 7, 534-549 (2004)

10. Payne, LE, Philippin, GA: Decay bounds for solutions of second order parabolic problems and their derivatives III. Z. Anal. Anwend. 23, 809-818 (2004)

11. Payne, LE, Philippin, GA, Vernier-Piro, S: Decay bounds for solutions of second order parabolic problems and their derivatives IV. Appl. Anal. 85, 293-302 (2006)

12. Philippin, GA, Vernier-Piro, S: Explicit exponential decay bounds in quasilinear parabolic problems. J. Inequal. Appl. 3 , 1-23 (1999)

13. Philippin, GA, Vernier-Piro, S: Explicit decay bounds in some quasilinear one-dimensional parabolic problems. Math Methods Appl. Sci. 22, 101-109 (1999)

14. Philippin, GA, Vernier-Piro, S: Decay estimates for solutions of a class of parabolic problems arising in filtration through porous media. Boll. Unione Mat. Ital. Sez. B Artic. Ric. Mat. (8) 4, 473-481 (2001)

15. Protter, MH, Weinberger, HF: Maximum Principles in Differential Equations. Prentice-Hall, Englewood Cliffs (1967)

16. Payne, LE, Philippin, GA: Decay bounds for solutions of second order parabolic problems and their derivatives. Math. Models Methods Appl. Sci. 5, 95-110 (1995)

17. Enache, C: Blow-up, global existence and exponential decay estimates for a class of quasilinear parabolic problems. Nonlinear Anal. TMA 69, 2864-2874 (2008)

18. Chen, SH: Global existence and blowup for quasilinear parabolic equations not in divergence form. J. Math. Anal. Appl. 401, 298-306 (2013)

19. Chen, SH, Yu, DM: Global existence and blowup solutions for quasilinear parabolic equations. J. Math. Anal. Appl. 335 151-167 (2007)

20. Ding, JT: Blow-up solutions for a class of nonlinear parabolic equations with Dirichlet boundary conditions. Nonlinear Anal. TMA 52, 1645-1654 (2003)

21. Payne, LE, Schaefer, PW: Lower bounds for blow-up time in parabolic problems under Dirichlet conditions. J. Math. Anal. Appl. 328, 1196-1205 (2007)

22. Wang, H, He, YJ: On blow-up of solutions for a semilinear parabolic equation involving variable source and positive initial energy. Appl. Math. Lett. 26, 1008-1012 (2013)

23. Payne, LE, Song, JC: Lower bounds for blow-up time in a nonlinear parabolic problem. J. Math. Anal. Appl. 354, 394-396 (2009)

24. $\mathrm{Xu}, \mathrm{RZ}, \mathrm{Cao}, \mathrm{XY}, \mathrm{Yu}$, T: Finite time blow-up and global solutions for a class of semilinear parabolic equations at high energy level. Nonlinear Anal., Real World Appl. 13, 197-202 (2012) 